\title{
Alveolar Epithelium
}

National Cancer Institute

\section{Source}

National Cancer Institute. Alveolar Epithelium. NCI Thesaurus. Code C12867.

Epithelia of lung alveoli. The layer of cells covering the lining of the tiny air sacs at the end of the bronchioles. 